\title{
Serviço farmacêutico: rastreamento da síndrome metabólica em servidores de uma Universidade Pública em Belém-PA
}

Pharmaceutical service: tracking of metabolic syndrome in servers of a Public University in BelémPA

\author{
Servicio farmacéutico: rastreo del síndrome metabólico en servidores de una Universidad Pública \\ en Belém-PA
}

Recebido: 09/10/2021 | Revisado: 18/10/2021 | Aceito: 26/10/2021 | Publicado: 30/10/2021

Priscila Danielle Moura da Costa

ORCID: https://orcid.org/0000-0002-1140-0152

Universidade Federal do Pará, Brasil

E-mail: priscilapjdanielle@gmail.com

Dhenyfer Andreza de Oliveira Gomes

ORCID: https://orcid.org/0000-0003-3590-5478 Universidade Federal do Pará, Brasil E-mail: da.gomes19@gmail.com

Taynah Isabelle Silva Soares

ORCID: https://orcid.org/0000-0002-0126-0010 Universidade Federal do Pará, Brasil E-mail: taynahisabelle@hotmail.com

Marcos Vinicius Soares Silva

ORCID: https://orcid.org/0000-0002-9687-1366 Universidade Federal do Pará, Brasil E-mail: mvsossil@gmail.com

Marcos Valério Santos da Silva ORCID: https://orcid.org/0000-0002-7824-0042 Universidade Federal do Pará, Brasil E-mail: marcossilva@ufpa.br

Marcieni Ataíde Andrade

ORCID: https://orcid.org/0000-0001-5875-695X Universidade Federal do Pará, Brasil E-mail: marcieni@ufpa.br

\begin{abstract}
Resumo
O objetivo deste estudo foi realizar o serviço farmacêutico de rastreamento em saúde da síndrome metabólica (SM) em servidores de uma Universidade Pública em Belém-PA. O estudo foi descritivo transversal, composto por servidores de ambos os sexos com idade entre 20 e 74 anos, a partir da coleta dos dados sociodemográficos, cardiometabólicos e aferição da circunferência abdominal (CA) para avaliação da obesidade central (OC). Caracterizou-se a SM pela agregação de pelo menos 3/5 componentes, que são: hiperglicemia em jejum, hipertrigliceridemia, hipolipidemia de lipoproteína de alta densidade (HDL-c), hipertensão arterial e OC. Para a análise estatística utilizou-se o software GraphPad Prism ${ }^{\circledR}$ - 5.00-Windows, com o Coeficiente de Correlação de Pearson para análise amostral; teste Quiquadrado para análise da presença/ausência da SM x estratos de Framingham; Teste Exato de Fisher para avaliar o risco relativo. Os resultados apontaram idade média de $46 \pm 13$ anos e a maioria de mulheres $(60,4 \%)$ dentro da amostra de 91 servidores. Houve predominância de níveis reduzidos de HDL-c (73,6\% e p=0,0165); de OC $(53,8 \%$ e p<0,0001) e de PA elevada (50,5\%). Entre os servidores 48,3\% foram classificados com "Presença de SM", dentre os quais houve predominância nos estratos médio $(69,2 \%)$ e alto $(82,6 \%)$. Já os classificados com "Ausência de SM" foram predominantes no estrato de baixo risco (70,9\%). As análises de risco cardiovascular x SM (p<0,0001) e idade x SM $(\mathrm{p}<0,0002)$, foram diretamente proporcionais. Percebeu-se um elevado percentual de servidores classificados com a "Presença de SM" e o rastreamento mostrou-se eficaz na detecção precoce dos componentes da SM.
\end{abstract}

Palavras-chave: Serviço farmacêutico; Rastreamento em saúde; Síndrome metabólica; Risco cardiovascular.

\begin{abstract}
The objective of this study was to realize the pharmaceutical service of health tracking of the metabolic syndrome (MS) in servers of a Public University in Belém-PA. The study was a cross-sectional descriptive, composed of servers of both sexes aged between 20 and 74 years, from the collection of sociodemographic and cardiometabolic data and measurement of abdominal circumference (AC) for evaluation of central obesity (CO). MS was characterized by the aggregation of at least $3 / 5$ components, which are: fasting hyperglycemia, hypertriglyceridemia, high-density
\end{abstract}


lipoprotein (HDL-c) hypolipidemia, arterial hypertension (AH) and CO. For the statistical analysis, the GraphPad Prism ${ }^{\circledR}$ - 5.00-Windows software was used, with Pearson's Correlation Coefficient for sample analysis; Chi-square test for analysis of the presence/absence of MS x Framingham strata; Fisher's Exact Test to evaluate the relative risk. The results showed a mean age of $46 \pm 13$ years and a majority of women $(60.4 \%)$ within the sample of 91 servers. There was a predominance of low HDL-c levels (73.6\% and $\mathrm{p}=0.0165)$; of $\mathrm{CO}$ (53.8\% and $\mathrm{p}<0.0001)$ and AH (50.5\%). Among the employees, $48.3 \%$ were classified as "Presence of MS", among which there was a predominance in the medium $(69.2 \%)$ and high $(82.6 \%)$ strata. Those classified as "Absence of MS" were predominant in the low-risk stratum $(70.9 \%)$. The analyses of cardiovascular risk x MS ( $\mathrm{p}<0.0001)$ and age $\mathrm{x}$ MS $(\mathrm{p}<0.0002)$ were directly proportional. A high percentage of servers classified as "Presence of MS" was perceived, and the screening proved to be effective in the early detection of the components of MS.

Keywords: Pharmaceutical servisse; Health tracking; Metabolic syndrome; Cardiovascular risk.

\section{Resumen}

El objetivo de este estudio fue realizar el servicio farmacéutico de rastreo en salud del síndrome metabólico (SM) en servidores de una Universidad Pública en Belém-PA. El estudio fue descriptivo transversal, compuesto por servidores de ambos sexos con edades comprendidas entre los 20 y los 74 años, a partir de la captación de datos sociodemográficos y cardiometabólicos y la medición de la circunferencia abdominal (CA) para la evaluación de la obesidad central (OC). El SM se caracterizó por la agregación de al menos 3/5 componentes, que son: hiperglucemia en ayunas, hipertrigliceridemia, hipolipidemia de lipoproteínas de alta densidad (HDL-c), hipertensión arterial (HA) y CA. Para el análisis estadístico se utilizó el software GraphPad Prism® - 5.00-Windows, con el coeficiente de correlación de Pearson para el análisis de la muestra; la prueba de Chi-cuadrado para analizar la presencia/ausencia de SM x estratos de Framingham; la prueba exacta de Fisher para evaluar el riesgo relativo. Los resultados indican una edad media de $46 \pm 13$ años y la mayoría de las mujeres $(60,4 \%)$ dentro del grupo de 91 servidores. Hubo un predominio de niveles reducidos de HDL-c $(73,6 \%$ y p=0,0165); de OC $(53,8 \%$ y p<0,0001) y de HA (50,5\%). El 48,3\% de los servidores fueron clasificados como "Presencia de SM", entre los que predominaban los estratos medio $(69,2 \%)$ y HA (82,6\%). Por otro lado, los clasificados como "Ausencia de SM" predominaban en el estrato de bajo riesgo (70,9\%). Los análisis de riesgo cardiovascular x SM(p<0,0001) y edad x SM ( $<<0,0002)$, fueron directamente proporcionales. Se observó un alto porcentaje de servidores clasificados como "Presencia de SM" y el cribado demostró ser eficaz en la detección temprana de los componentes de la SM.

Palabras clave: Servicio farmacéutico; Rastreo en salud; Síndrome metabólico; Riesgo cardiovascular.

\section{Introdução}

Ao longo dos anos, ocorreu na sociedade diversas transformações no padrão demográfico, epidemiológico, nutricional e comportamental, refletindo na saúde da população mundial, e elevando a incidência da morbimortalidade associada às doenças crônicas não transmissíveis (DCNTs), como dislipidemia, hipertensão arterial sistêmica (HAS), e diabetes mellitus tipo 2 (DM2) definidas como fatores de risco para doenças cardiovasculares (DCVs) (Cazarim et al., 2016). Estes fatores de risco, associados à obesidade central (OC) em um único indivíduo constituem a Síndrome Metabólica (SM) (Kaur, 2014; Soares et al., 2014). A SM não pode ser qualificada como uma doença singular, pois abrange diversos componentes de origem metabólica e hemodinâmica, propensos à agregação. Todavia, generalizar a classificação desta síndrome compreende dificuldades, visto que há uma variação entre os elementos para o seu diagnóstico que dependem do lugar de origem, do gênero, de atributos étnicos, de características físicas, de hábitos alimentares e de estilo de vida (Junqueira et al., 2011; Associação Brasileira para o Estudo da Obesidade e da Síndrome Metabólica [Abeso], 2016).

A Organização Mundial de Saúde (OMS), em 1998 definiu a SM com critérios baseados na elevação do índice glicêmico, tendo como principal elemento, a resistência à insulina, sendo por tanto uma definição glucocêntrica (Azambuja et al., 2015), e que poderia subestimar a prevalência e incidência da SM em populações mais carentes. Posteriormente, em 2001, o National Cholesterol Education Program - Adult Treatment Panel III (NCEP-ATP III) estabeleceu novos componentes para o diagnóstico da SM, de modo que as alterações glicêmicas deixaram de ser consideradas um fator indispensável, tornando-se apenas, um dos elementos constituintes para o diagnóstico desta síndrome. O NCEP- ATP III, classifica a SM pela agregação de no mínimo 3 dos 5 componentes, que são relacionados com a elevação ou redução de seus parâmetros, ou seja, níveis elevados da glicose em jejum, dos triglicerídeos (TG), da pressão arterial (PA) e da CA para mensurar a OC e baixos níveis de HDL-c (Ford, 2005; Abeso, 2016). Estes critérios foram adotados pela I Diretriz Brasileira de Diagnóstico e Tratamento da Síndrome 
Metabólica (DBSM), que também recomendou como critérios específicos para o diagnóstico da SM o tratamento farmacológico para HAS e dislipidemias, e detecção precoce de DM2 (Carvalho, 2005; Rubbo-Blanco et al., 2015).

A alta prevalência da SM é um fator preocupante, pois suas complicações crônicas oriundas do impacto causado por seus componentes, interferem diretamente na qualidade de vida da sociedade, caracterizando-se como um grande problema de saúde pública, deixando em alerta a comunidade científica e multiprofissional em saúde (Simão et al., 2013; Soares et al., 2014). Todavia, ainda existe uma lacuna na literatura sobre a prevalência da SM e estima-se que mundialmente, em adultos, esteja entre 20 e 25\%, e no Brasil entre adultos e idosos, o percentual encontrado é 9\% (Leitão \& Martins, 2012). Nos indivíduos com SM, o risco relativo de desenvolver DCVs varia entre 1,53 a 2,18 e o índice de mortalidade é elevado em 2,5 vezes (Carvalho, 2005; Simão et al., 2013). Comumente, a SM tem sido detectada tardiamente, situações nas quais apresentam manifestações da DM2 e agravos cardiovasculares. Sendo assim, é primordial a adoção de estratégias de prevenção que busquem reduzir a incidência e as complicações causadas pela SM. Deste modo, o manejo da SM necessita de ações de promoção à saúde e prevenção, pois a identificação precoce dos fatores de risco que a compõem é fundamental para minimizar seus danos (Cordero et al., 2013).

O rastreamento em saúde é um serviço que permite a possível detecção precoce de uma doença ou distúrbio de saúde em indivíduos assintomáticos ou com risco de desenvolvê-las. Ocorre por meio de exames e testes ou do uso de questionários validados. Os resultados são avaliados para adoção da conduta clínica mais adequada em relação à situação de saúde do indivíduo que será orientado e, se necessário, encaminhado a outro profissional ou instituição de saúde buscando elucidar a hipótese diagnóstica e o tratamento assertivo (Conselho Federal de Farmácia [CFF], 2016). Diversos profissionais de saúde são habilitados a realizar este serviço, e os processos utilizados para fazê-lo devem ser econômicos, com boa acurácia e reprodutibilidade, de aplicação simples e o menos invasivo possível para que sejam aceitos (Ministério da Saúde [MS], 2010; Précoma et al., 2019).

Um programa de rastreamento em saúde quando bem elaborado e executado com excelência assegura ao cidadão o direito à atenção à saúde de qualidade, pois não há necessidade da solicitação de outro profissional de saúde para a realização de um exame ou procedimento presente no rastreamento, devido não se tratar de diagnóstico de um quadro clínico, mas sim de critérios definidos que permitem ao indivíduo participar das ações (MS, 2010; CFF, 2016). No sistema de saúde a crescente oferta deste serviço poderia cooperar para minimizar o subdiagnóstico médico e aumentar a prevenção de diversas doenças, como as DCVs e seus fatores de risco que quando agregados, constituem a SM (Peeling \& Ye, 2004; Cordero et al, 2013).

O serviço de rastreamento em saúde de diversas doenças, principalmente em indivíduos assintomáticos, está contemplado dentro da prática do cuidado farmacêutico. De modo que, este profissional torna-se um agente que pode atuar na prevenção do desenvolvimento de eventos cardiovasculares. Visto que os serviços farmacêuticos são definidos como um agrupamento de atividades instituídas em um processo de trabalho, buscando corroborar para prevenção de doenças, promoção, proteção e recuperação da saúde e auxiliar para a melhoria da qualidade de vida dos indivíduos (CFF, 2016).

Segundo Valls e Fernandez-Llimos (2005 como citado em CFF, 2016, p.76) o farmacêutico por meio da prestação de serviços farmacêuticos tem posição estratégica em sua atuação profissional por ter contato diário com uma ampla quantidade de indivíduos assintomáticos sob o risco de desenvolver algumas doenças e pode cooperar para a oferta do serviço de rastreamento em saúde, de modo habitual em diversos cenários, e contribuir para a identificação de pessoas com alto risco em desenvolver DCVs, mediante deteç̧ão de seus fatores de risco (Malachias et al., 2016; Tsuyuki et al., 2016).

As universidades apesar de serem locais de amplo desenvolvimento de pesquisas enfrentam desafios para desenvolverem e implantarem ações em saúde significativas de prevenção de doenças, bem como para a promoção da saúde dos trabalhadores universitários, visto que estes não são incluídos frequentemente em propósitos de pesquisas, e sim a comunidade que recebe atendimento em hospitais, clínicas, laboratórios, farmácias universitárias entre outros. Outrossim, trabalhadores que permanecem grande parte do seu dia exercendo suas atividades laborais, não recebem a atenção a saúde necessária e nem os benefícios dos serviços, programas e projetos em saúde ofertados por essas instituições. Logo este ambiente universitário torna- 
se propício para o desenvolvimento de serviços farmacêuticos como o rastreamento em saúde voltado para os trabalhadores universitários.

Dessa forma, este trabalho teve por objetivo realizar o serviço farmacêutico de rastreamento em saúde da síndrome metabólica e de seus componentes em servidores de uma Universidade Pública, visto que, a detecção precoce destes fatores é condição essencial para a prevenção de distúrbios metabólicos e de doenças ateroscleróticas.

\section{Metodologia}

Trata-se de estudo descritivo transversal com análise quantitativa, derivado do projeto de mestrado intitulado “CONSULTÓRIO FARMACÊUTICO COMO DISPOSITIVO ASSISTENCIAL EM SAÚDE PARA AVALIAÇÃO DO RISCO CARDIOVASCULAR DOS SERVIDORES DE UMA INSTITUIÇÃO PÚBLICA DE ENSINO SUPERIOR”, realizado em Belém-PA, com servidores técnicos da Universidade Federal do Pará. Este estudo foi aprovado pelo Comitê de Ética e Pesquisa em Seres Humanos da Universidade Federal do Pará sob o protocolo CAAE: 98166818.90000.0018.

A amostragem foi probabilística aleatória simples, composta por servidores técnicos de ambos os sexos. Inicialmente 177 servidores atenderam aos critérios de inclusão que foram: ser servidor técnico ativo ou inativo, com idade entre 20 e 74 anos, que concordaram e assinaram o Termo de Consentimento Livre e Esclarecido (TCLE). Foram excluídos os servidores docentes e/ou pesquisadores, servidores com distúrbios mentais ou cognitivos, servidores com evento cardiovascular prévio não fatal e servidora gestante. Entretanto, nem todos os participantes inicialmente incluídos realizaram todas as etapas do estudo. Os dados foram coletados no período de setembro/2019 a março/2020 por estudantes do curso de Farmácia previamente selecionados e capacitados.

Os servidores foram convidados a participar do estudo por meio de campanhas por intermédio de ações de rastreamento em saúde desenvolvidas no campus profissional e básico da UFPA, onde foram informados sobre a futura realização da consulta farmacêutica e exames laboratoriais que foram realizados no Ambulatório de Cuidados Farmacêuticos/Laboratório de Análises Clínicas e no Laboratório de Hematologia Clínica, ambos na Faculdade de Farmácia na referida Universidade. O agendamento da consulta farmacêutica ocorreu posteriormente as ações por contato via E-mail ou mensagem de WhatsApp ou ligação telefônica.

Durante a consulta farmacêutica o servidor foi submetido a uma entrevista, na qual respondeu um questionário sociodemográfico e de histórico de saúde, além da aferição da PA e de medidas antropométricas. Também, foi agendada a coleta de sangue para obtenção das variáveis metabólicas (glicemia em jejum, HDL-c, colesterol total e TG) e os servidores foram orientados quanto a necessidade de jejum prévio de 10 a 12 horas para a realização desses exames (Faludi et al., 2017; Golbert et al., 2017). O questionário foi constituído por perguntas referentes à idade, sexo, etnia, escolaridade, renda individual mensal, presença de HAS, de DM2 e de dislipidemia e quanto ao uso de medicamentos para o tratamento destas doenças quando previamente diagnosticadas.

A variável antropométrica utilizada neste estudo foi a CA para mensuração da OC. Para obtenção da CA foi utilizada uma fita métrica inextensível colocada acima da cicatriz umbilical com o servidor em pé, ereto, com o abdômen relaxado, braços estendidos ao longo do corpo, e pernas paralelas ligeiramente separadas. A OC foi observada nos servidores com CA com valores $\geq 88 \mathrm{~cm}$ para mulheres e $\geq 102 \mathrm{~cm}$ para homens (Carvalho, 2005; MS, 2011; Abeso, 2016).

A PA foi aferida conforme o preconizado na $7^{\circ}$ Diretriz Brasileira de Hipertensão Arterial. O procedimento foi realizado com esfigmomanômetro aneroide e estetoscópio convencional da NTL Pressure ${ }^{\circ}$, previamente calibrados, após 15 minutos de repouso. Os níveis pressóricos foram considerados elevados quando $\geq 130 \mathrm{mmHg}$ e/ou $85 \geq \mathrm{mmHg}$ (Carvalho, 2005; Malachias et al., 2016). 
Para a realização dos exames laboratoriais da glicemia em jejum e do perfil lipídico (TG, HDL-c e colesterol total) os servidores relataram jejum prévio de 10 a 12 horas. Os kits enzimáticos utilizados foram da Labtest Diagnóstica ${ }^{\circledR}$ e o analisador o Microlab 300 (ELITech Clinical Systems SAS), as análises foram feitas conforme as instruções dos fabricantes. Considerouse resultados inadequados: glicemia em jejum $\geq 100 \mathrm{mg} / \mathrm{dL}$; triglicérides $\geq 150 \mathrm{mg} / \mathrm{dL}$; HDL-C $<50 \mathrm{mg} / \mathrm{dL}$ em mulheres e $<40$ $\mathrm{mg} / \mathrm{dL}$ em homens, e colesterol total $\geq 190 \mathrm{mg} / \mathrm{dL}$ (Faludi et al., 2017; Golbert et al., 2017). A medida do colesterol total foi utilizada apenas na estratificação do risco cardiovascular pelo escore de risco de Framingham (Précoma et al., 2019). Os demais parâmetros foram utilizados para o rastreamento da SM (Carvalho, 2005; Abeso, 2016).

A estratificação do risco cardiovascular dos servidores foi feita com a aplicação do escore de risco de Framingham (ERF), que utiliza como preditores sexo, idade, PAS tratada ou não tratada, tabagismo, DM2 e perfil lipídico (HDL-c e CT). Cada preditor foi pontuado e somado, e aos resultados, quando necessário, foi aplicado o fator de correção. Posteriormente o resultado da adição foi correlacionado ao percentual de estratificação de risco de desenvolver um evento cardiovascular em 10 anos. Os percentuais, foram classificados como: risco baixo para $<10 \%$, risco médio para $>10 \%$ e $<20 \%$ e risco alto para $>$ 20\%. A estratificação foi realizada nas planilhas de Excel disponibilizadas na página do Estudo do Coração de Framingham (Framingham Heart Study) (MS, 2010; Précoma et al., 2019).

Os critérios adotados para o rastreamento da SM seguem o preconizado pela I DBSM, e pelo NCEP-ATP III. Deste modo, a SM é definida quando da agregação de no mínimo 3 dos seguintes componentes: glicose em jejum $\geq 100 \mathrm{mg} / \mathrm{dL}$ ou quando diagnóstico prévio de DM 2; HDL-c para homens $<40 \mathrm{mg} / \mathrm{dL}$ e para mulheres $<50 \mathrm{mg} / \mathrm{dL}$; triglicerídeos $\geq 150 \mathrm{mg} / \mathrm{dL}$; níveis pressóricos (PAS e/ou PAD) $\geq 130 \mathrm{mmHg}$ e/ou $\geq 85 \mathrm{mmHg}$, ou respectivamente quando da utilização de medicamentos para dislipidemias e HAS. Além da OC, verificada por meio da circunferência abdominal, para homens $\geq 102 \mathrm{~cm}$ e para mulheres $\geq 88 \mathrm{~cm}$ (Ford, 2005; Carvalho, 2005; Abeso, 2016). A classificação "Presença de SM" foi identificada no servidor que apresentou a agregação de no mínimo 3 componentes desta síndrome, enquanto a denominação "Ausência de SM" foi atribuída para aquele servidor que não apresentou nenhum componente ou aquele que apresentou a agregação de até 2 componentes.

A tabulação dos dados foi realizada em planilhas no programa Microsoft Excel ${ }^{\circledR}$ 2019. Foi usado o software GraphPad Prism ${ }^{\circledR}$ versão 5.00 para Windows para a análise descritiva no qual foram calculados média aritmética, desvio-padrão, frequência absoluta e relativa. Além dos valores máximo e mínimo para a idade. Já para as análises estatísticas, os dados paramétricos, quando possível, foram correlacionados e sua significância foi analisada com o Coeficiente de Correlação de Pearson (r) para análise amostral. Para a análise da correlação da presença da SM com os estratos de risco cardiovascular e das faixas etárias com a presença e ausência da SM, foi aplicado o teste Qui-quadrado ( $\chi 2)$. O risco relativo para correlacionar a presença e ausência de SM e o risco cardiovascular subdividido em baixo e médio/alto foi verificado através do Teste Exato de Fisher. A significância estatística estabelecida foi de $\mathrm{p}<0,05$, com intervalo de confiança de $95 \%$.

\section{Resultados}

Noventa e um servidores de ambos os sexos foram avaliados, sendo 60,4\% (55) do sexo feminino. A idade mínima e máxima foram respectivamente de 24 e 71 anos, com média e desvio padrão de $46 \pm 13$ anos, 44\% (40) dos servidores estavam na faixa etária de 41 a 60 anos, e 40,6\% (37) tinham idade entre 20 e 40 anos. Quanto à escolaridade, 48,4\% (44) eram pósgraduados e $45 \%$ (41) eram graduados, 58,2\% (53) autorrelataram ter cor de pele parda e a renda individual mensal para 53,8\% (49) era igual ou maior a 5 salários mínimos (Tabela 1). 
Tabela 1 - Perfil sociodemográfico dos servidores de uma universidade pública em Belém-PA, 2020.

\begin{tabular}{lr}
\hline Variáveis sociodemográficas & $\mathrm{n} 91(100 \%)$ \\
\hline Sexo & $55(60,4 \%)$ \\
Feminino & $36(39,6 \%)$ \\
Masculino & \\
\hline Idade (anos) & $37(40,6 \%)$ \\
$20-40$ & $40(44 \%)$ \\
$41-60$ & $14(15,4 \%)$ \\
$>60$ & \\
Escolaridade & $4(4,4 \%)$ \\
Médio Completo & $2(2,2 \%)$ \\
Superior Incompleto & $41(45 \%)$ \\
Superior Completo & $44(48,4 \%)$ \\
Pós-Graduado & \\
\hline Etnia* & $16(17,6 \%)$ \\
Preto & $53(58,2 \%)$ \\
Pardo & $22(24,2 \%)$ \\
Branco & \\
\hline Renda Mensal - Sm & $42(46,2 \%)$ \\
$1-4$ & $49(53,8 \%)$ \\
\hline 5 &
\end{tabular}

n- número total da amostra; Sm - salário mínimo; *etnias indígena /asiática, não foram autorrelatadas.

Fonte: Autores.

Dos componentes da SM, na amostra estudada, a maior prevalência foi do HDL-c baixo, presente em 73,6\% (67), dos servidores, seguido pela presença de OC em 53,8\% (48) e elevação de PAS e/ou PAD em 50,5\% (46) deles. Na análise amostral, houve diferença significativa apenas em relação ao HDL-c, p =0,0165 e a OC, p<0,0001. Na análise entre os sexos, somente a PA foi mais prevalente entre os homens 55,6\% (20) do que entre as mulheres 47,3\% (26). A OC 58,2\% (32), e o HDL-c baixo $76,4 \%$ (42) tiveram mais prevalência entre as mulheres quando comparadas aos homens (Tabela 2).

Neste estudo, 25,3\% (23) dos servidores apresentaram 3 componentes agregados para SM, 18,7\% (17) apresentaram 4, e 4,4\% (4) continham a agregação de todos os componentes desta síndrome, por isso todos foram classificados com a "Presença de SM". Entre aqueles classificados em "Ausência de SM" o percentual de servidores que não apresentavam nenhum componente foi de 7,7\% (7), daqueles com apenas um componente foi de 17,6\% (16) e dos com agregação de dois componentes foi de $26,4 \%$ (24). Quando a análise foi conforme o sexo verificou-se que entre as mulheres 27,3\% (15) continham 2 componentes associados e 23,6\% (13) delas, possuíam igualmente a associação de 3 e 4 componentes. Já entre os homens 27,8\% (10) apresentaram a agregação de 3 componentes e $25 \%$ (9) reuniram 2 componentes agregados (Tabela 2).

$\mathrm{Na}$ amostra a classificação "Presença de SM" foi prevalente em 48,3\% (44) dos servidores, porém sem significância estatística. Quando se verificou a prevalência da classificação "Presença de SM" de acordo com o sexo, percebeu-se um percentual aproximado entre homens e mulheres (Tabela 2). Conforme a classificação adotada neste estudo, de modo geral entre os classificados com "Ausência de SM" a prevalência foi de 51,6\%. Nas mulheres esta prevalência foi de 50,9\%, e nos homens de $52,8 \%$ (dados não mostrados). 
Tabela 2 - Prevalência dos componentes da síndrome metabólica, do número de componentes agregados, e da classificação "Presença da SM", de forma geral e segundo o sexo em servidores de uma universidade pública em Belém-PA, 2020.

\begin{tabular}{|c|c|c|c|c|c|}
\hline \multirow{3}{*}{ Componentes da SM } & \multicolumn{2}{|c|}{ Sexo } & \multirow{2}{*}{\multicolumn{2}{|c|}{ Total }} & \multirow{3}{*}{ p - Valor ${ }^{r}$} \\
\hline & \multirow{2}{*}{$\begin{array}{c}\text { Feminino } \\
\mathrm{n}(\%)\end{array}$} & \multirow{2}{*}{$\begin{array}{c}\text { Masculino } \\
\mathrm{n}(\%)\end{array}$} & & & \\
\hline & & & $\mathrm{n}(\%)$ & (IC 95\%) & \\
\hline Glicemia em jejum $\geq 100 \mathrm{mg} / \mathrm{dL}^{\mathrm{a}}$ & $15(27,3)$ & $9(25)$ & $24(26,4)$ & $(-0,2300 ; 0,1817)$ & 0,8124 \\
\hline Pressão arterial $\geq 130 / 85 \mathrm{mmHg}^{\mathrm{b}}$ & $26(47,3)$ & $20(55,6)$ & $46(50,5)$ & $(-0,1271 ; 0,2823)$ & 0,4453 \\
\hline $\begin{array}{l}\text { Obesidade central, circunferência } \\
\text { abdominal } \geq 102 \mathrm{~cm} \text { homens } \mathrm{e} \geq 88 \mathrm{~cm} \\
\text { mulheres }\end{array}$ & $32(58,2)$ & $17(47,2)$ & $49(53,8)$ & $(0,2203 ; 0,5662)$ & $<0.0001$ \\
\hline $\begin{array}{l}\text { Lipoproteína de alta densidade, homens } \\
<40 \mathrm{mg} / \mathrm{dL} \text { e mulheres }<50 \mathrm{mg} / \mathrm{dL}^{c}\end{array}$ & $42(76,4)$ & $25(69,4)$ & $67(73,6)$ & $(-0,4343 ; 0,04713)$ & 0,0165 \\
\hline Triglicerídeo, $\geq 150 \mathrm{mg} / \mathrm{dL}^{\mathrm{c}}$ & $21(38,2)$ & $14(38,9)$ & $35(38,5)$ & $(-0,2365 ; 0,1751)$ & 0,7627 \\
\hline Número de componentes agregados & & & & & \\
\hline 0 & $3(5,5)$ & $4(11,1)$ & $7(7,7)$ & & \\
\hline 1 & $10(18,2)$ & $6(16,7)$ & $16(17,6)$ & & \\
\hline 2 & $15(27,3)$ & $9(25)$ & $24(26,4)$ & & \\
\hline 3 & $13(23,6)$ & $10(27,8)$ & $23(25,3)$ & & \\
\hline 4 & $13(23,6)$ & $4(11,1)$ & $17(18,7)$ & & \\
\hline 5 & $1(1,8)$ & $3(8,3)$ & $4(4,4)$ & & \\
\hline *Síndrome Metabólica & $27(49,1)$ & $17(47,2)$ & $44(48,3)$ & $(-0,2234 ; 0,1884)$ & 0,8634 \\
\hline
\end{tabular}

a- ou no diagnóstico pregresso de DM2; b- ou no tratamento para HAS; c- ou no tratamento para dislipidemias;

r- Coeficiente de Correlação de Pearson, $\mathrm{p}<0,05$, com intervalo de confiança de $95 \%$.

*Nesta análise adotou-se os critérios definidos pela I DBSM e pelo NCEP-ATP III, que considera a agregação de pelo menos 3 componentes em um único indivíduo para classificar a presença de SM.

Fonte: Autores.

O risco cardiovascular dos servidores foi avaliado pelo escore de risco de Framingham (Figura1).

Figura 1 - Prevalência da estratificação do risco cardiovascular pelo escore de Framingham, em análise geral e conforme o sexo em servidores de uma universidade pública em Belém-PA, 2020.

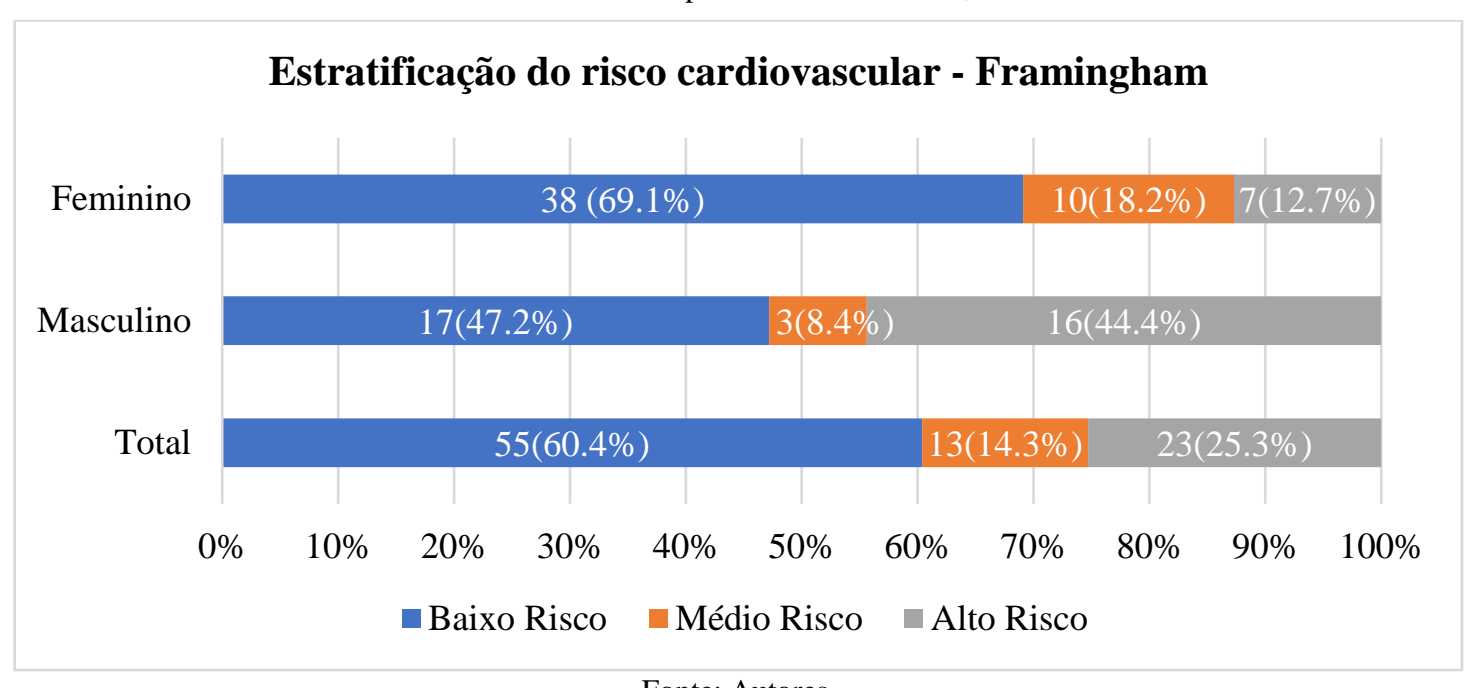

Fonte: Autores.

A estratificação de risco cardiovascular pelo escore de risco de Framingham mostrou que 60,4\% (55) dos servidores foram estratificados em baixo risco cardiovascular, e 25,3\% (23) foram estratificados como alto risco de desenvolver um evento cardiovascular em 10 anos. O risco cardiovascular foi baixo para a maioria dos homens e das mulheres, respectivamente, em 
$47,2 \%$ (17) e $69,1 \%$ (38). Contudo, 44,4\% (16) dos homens foram alocados em alto risco cardiovascular e apenas $12,7 \%$ (7) das mulheres estavam neste estrato (Figura 1).

A avaliação da relação dos estratos de risco cardiovascular do ERF com a classificação com "Presença de SM" e com "Ausência de SM" nos servidores foi exposta na Tabela 3.

Tabela 3 - Distribuição dos estratos do Escore de risco de Framingham, relacionados a com "Presença de SM" e com "Ausência de SM" em análise geral e conforme o sexo em servidores de uma universidade pública em Belém-PA, 2020.

\begin{tabular}{lcccc}
\hline & & \multicolumn{2}{c}{ Classificação segundo a I DBSM e o } & \\
\cline { 3 - 4 } ER - ERF & Total & \multicolumn{2}{c}{ NCEP-ATP III" } & \multirow{2}{*}{ p-Valor } \\
& $91(100 \%)$ & "Presença de SM" & "Ausência de SM" & \\
\hline Risco Baixo & 55 & $16(29,4 \%)$ & $47(51,6 \%)$ & \\
Risco Médio & 13 & $9(69,2 \%)$ & $39(70,9 \%)$ & \\
Risco Alto & 23 & $19(82,6 \%)$ & $4(30,8 \%)$ & $<0,0001^{\text {a; b }}$ \\
\hline
\end{tabular}

ER-ERF- Estratificação de risco pelo escore de risco de Framingham; SM- síndrome metabólica. a- Teste Qui-quadrado; b- Teste Exato de Fisher; significância quando p <0,05.

*Nesta análise adotou-se os critérios definidos pela I DBSM e pelo NCEP-ATP III, que considera a agregação de pelo menos 3 componentes em um único indivíduo para classificar a presença de SM.

Fonte: Autores.

Ao relacionar os estratos do ERF com aqueles servidores classificados em "Presença de SM" e em "Ausência de SM", foi verificado que no estrato de baixo risco 70,9\% (39) apresentaram agregação de no máximo 2 componentes da SM, sendo classificados em "Ausência de SM". Todavia, nos estratos de médio e alto risco, a maioria dos servidores, respectivamente, 69,2\% (9) e 82,6\% (19), foram classificados em "Presença de SM". Na análise do risco cardiovascular com a presença ou ausência de SM houve diferença significativa $(\mathrm{p}<0,0001)$, assim como na correlação daqueles classificados em Presença/Ausência de SM versus o risco cardiovascular subdividido em baixo e médio/alto ( $\mathrm{p}<0,0001)$. O risco relativo foi de 3,191(Tabela 3).

A análise das faixas etárias mostrou que nos servidores com idade entre 20 e 40 anos, o HDL-c baixo apareceu em $62,2 \%$ (23) deles. Já entre aqueles com idade de 41 a 60 anos, percebeu-se que 62,5\% (25), 65\% (26) e 80\% (30) apresentaram respectivamente, PA elevada, obesidade central e HDL-c baixo, e 65\% (26) foram classificados em "Presença de SM". No grupo etário > 60 anos, 85,7\% (12) apresentaram a PA elevada, 64,3\% (9) estavam obesos, e 85,7\% (12) tiveram o HDL-C baixo. Entre eles 78,6\% (11) foram classificados em "Presença de SM". Houve associação significante entre a idade e a classificação "Presença de SM" (p=0,0002) (Tabela 4).

Quanto à escolaridade, a hiperglicemia teve maior prevalência entre aqueles com nível superior incompleto 50\% (1). A PA estava elevada em todos com ensino médio completo e superior incompleto, $100 \%$ (3) e 100\% (2) respectivamente, e em $51,2 \%$ (21) dos graduados. A obesidade central teve maior frequência entre aqueles com a graduação completa 56,1\% (23) e nos pós-graduados 56,8\% (25). O HDL-c baixo foi observado em 66,7\% (2), 100\% (2), 73,2\% (30) e 72,7\% (32) daqueles com ensino médio completo, superior incompleto, superior completo e pós-graduados, respectivamente. Em 33,3\% (1) com ensino médio completo, 36,6\% (15) dos graduados, e em 40,9\% (18) dos pós-graduados o TG estava elevado. A classificação “Presença de SM" foi para, 50\% (1) dos graduandos, 66,7\% (2) com ensino médio e 51,2\% (21) dos graduados (Tabela 4).

A raça/etnia foi autorrelatada pelos servidores, entre os de pele preta 68,7\% (11), 50\% (8), e 81,2\% (13), apresentaram respectivamente, PA elevado, OC, e HDL-c baixo, nos quais a classificação em "Presença de SM" foi verificada em 68,7\% (11). Para os pardos o HDL-c baixo teve maior prevalência 75,5\% (40) entre os componentes da SM. Destes, 43,4\% (23) foram classificados em "Presença de SM. Entre os de pele branca 59,1\% (13), 68,2\% (15), 63,6\% (14), estavam respectivamente com 
a PA elevada, eram considerados obesos, e com HDL-c baixo, 45,4\% (10) foram classificados em "Presença de SM. Vale ressaltar que houve maior prevalência de níveis pressóricos elevados entre os de cor de pele preta (Tabela 4).

A renda mensal dos servidores teve como base o salário mínimo $(\mathrm{Sm})$, entre os que relataram que seus vencimentos variaram de 1 a 4 Sm, o HDL-c baixo foi o componente da SM mais prevalente, em 71,4\% (30) e 35,7\% (15) deles foram classificados em e "Presença de SM". Os que apresentaram renda mensal igual ou superior a $5 \mathrm{Sm}$, em 55,1\% (27), 61,2\% (30), e 75,5\% (37), respectivamente, a PA estava elevada, eram obesos, e o HDL-c estava baixo. A classificação em "Presença de SM" foi para 59,2\% (29) deles (Tabela 4).

Tabela 4 - Distribuição dos componentes da SM e da classificação "Presença de SM" quando relacionados com os dados sociodemográficos, em servidores de uma instituição pública de ensino superior em Belém - PA, 2020.

\begin{tabular}{|c|c|c|c|c|c|c|}
\hline & \multicolumn{5}{|c|}{ Componentes da SM (I DBSM - NCEP - ATP III) } & \multirow[b]{2}{*}{$\begin{array}{c}\text { SM } \\
\mathrm{n}(\%)\end{array}$} \\
\hline & $\begin{array}{c}\text { GJ elevada } \\
\mathrm{n}(\%)\end{array}$ & $\begin{array}{c}\text { PA elevada } \\
\mathrm{n}(\%)\end{array}$ & $\begin{array}{c}\text { OC } \\
\mathrm{n}(\%)\end{array}$ & $\begin{array}{c}\text { HDL-c baixo } \\
\text { n }(\%)\end{array}$ & $\begin{array}{c}\text { TG elevado } \\
\mathrm{n}(\%)\end{array}$ & \\
\hline Idade & & & & & & $\mathrm{p}=0,0002^{\mathrm{a}}$ \\
\hline $20-40(37)$ & $2(5,4)$ & $9(24,3)$ & $14(37,8)$ & $23(62,2)$ & $11(29,7)$ & $7(18,9)$ \\
\hline $41-60(40)$ & $15(37,5)$ & $25(62,5)$ & $26(65)$ & $32(80)$ & $17(42,5)$ & $26(65)$ \\
\hline$>60(14)$ & $7(50)$ & $12(85,7)$ & $9(64,3)$ & $12(85,7)$ & $7(50)$ & $11(78,6)$ \\
\hline \multicolumn{7}{|l|}{ Escolaridade } \\
\hline $\mathrm{MC}(3)$ & $1(33,3)$ & $3(100)$ & $1(33,3)$ & $2(66,7)$ & $1(33,3)$ & $2(66,7)$ \\
\hline SI (2) & $1(50)$ & $2(100)$ & 0 & $2(100)$ & 0 & $1(50)$ \\
\hline $\mathrm{SC}(41)$ & $10(24,4)$ & $21(51,2)$ & $23(56,1)$ & $30(73,2)$ & $15(36,6)$ & $21(51,2)$ \\
\hline PG (44) & $12(27,3)$ & $20(45,4)$ & $25(56,8)$ & $32(72,7)$ & $18(40,9)$ & $20(45,4)$ \\
\hline \multicolumn{7}{|l|}{ Raça/Etnia $^{+}$} \\
\hline Preto (16) & $7(43,7)$ & $11(68,7)$ & $8(50)$ & $13(81,2)$ & $6(37,5)$ & $11(68,7)$ \\
\hline Pardo (53) & $13(24,5)$ & $22(41,5)$ & $26(49)$ & $40(75,5)$ & $20(37,7)$ & $23(43,4)$ \\
\hline Branco (22) & $4(18,2)$ & $13(59,1)$ & $15(68,2)$ & $14(63,6)$ & $9(40,9)$ & $10(45,4)$ \\
\hline \multicolumn{7}{|l|}{ RM-Sm } \\
\hline $1-4(42)$ & $8(19)$ & $19(45,2)$ & $19(45,2)$ & $30(71,4)$ & $11(26,2)$ & $15(35,7)$ \\
\hline$\geq 5(49)$ & $16(32,6)$ & $27(55,1)$ & $30(61,2)$ & $37(75,5)$ & $24(48,9)$ & $29(59,2)$ \\
\hline
\end{tabular}

GJ- Glicemia em jejum; PA - pressão arterial; OC - obesidade central; HDL-c - lipoproteína de alta densidade; TG - triglicerídeos; SM - Síndrome Metabólica; MC - Médio completo; SI - Superior incompleto; SC - Superior completo; PG - pós-graduação; RM renda mensal; Sm - salário mínimo.

+ Não houve na amostra estudada, autorrelato das etnias indígena e asiática; a - Teste Qui-quadrado p <0,05.

*Nesta análise adotou-se os critérios definidos pela I DBSM e pelo NCEP-ATP III, que considera a agregação de pelo menos 3 componentes em um único indivíduo para classificar a presença de SM.

Fonte: Autores.

\section{Discussão}

Este estudo buscou identificar a prevalência dos componentes da SM e da "Presença de SM" por meio da prestação do serviço farmacêutico de rastreamento em saúde em servidores técnicos universitários, assim como relacioná-las aos estratos do ERF.

No âmbito da profissão farmacêutica o diagnóstico não está incluído no arcabouço de suas atribuições clínicas. Para atuar na identificação precoce das comorbidades crônicas que compõem a SM é ofertado o serviço farmacêutico de rastreamento em saúde (Carvalho, 2005; CFF, 2016). Quando realizado por este profissional mesmo que ainda esteja em processo de desenvolvimento, o rastreamento em saúde, mostra ter potencialidade de evolução e pode gerar trabalhos futuros que tratem sobre seu impacto financeiro, clínico e humanista (Ambiel \& Mastroianni, 2013; CFF, 2016). Sob esta perspectiva, o farmacêutico atua no processo de promoção à saúde, incentivando a adesão farmacoterapêutica e, portanto, melhorando os resultados, no controle e na prevenção de agravos em saúde em grupos de pacientes com vários tipos de doenças, entre as quais as DCNTs como DM2, HAS e dislipidemias, que quando associadas a outros fatores de risco a saúde maximizam o avanço da 
SM e elevam o risco de desenvolvimento de eventos cardiovasculares (MS, 2010; CFF,2016; Cazarim et al., 2016; Soares, et al., 2016; Barros et al., 2019).

A SM tem sua fisiopatologia ligada a uma condição de inflamação crônica de baixo grau devido às inter-relações entre os componentes metabólicos, comportamentais e genéticos (Ferreira et al, 2013; Kaur, 2014), e tem íntima associação a números expressivos de complicações cardiovasculares fatais e não fatais. Sendo um tema de relevância mundial importante para o desenvolvimento de diversas pesquisas. Entretanto, torna-se difícil estabelecer um estudo epidemiológico atualizado em virtude da falta de métodos padronizados. Por isso, são adotadas medidas que mensuram a prevalência desta síndrome com base na padronização dos critérios de risco dificultando a correlação dos achados deste estudo com os demais (Junqueira et al., 2011).

O perfil sociodemográfico na amostra de estudo mostrou que houve maior proporção de mulheres, a faixa etária mais prevalente foi a intermediária, a maioria tinha nível superior ou nível superior e pós-graduação, se definiram como pardos e apresentavam renda mensal igual ou maior que 5 salários mínimos (Sm). Ito et al. (2014), buscaram avaliar os fatores de risco cardiovasculares e a prevalência da SM em seu estudo com servidores da Universidade Estadual de Ponta Grossa (UEPG), onde também verificaram maior frequência de mulheres. O mesmo padrão foi percebido por Oliveira et al. (2020) ao verificarem a prevalência da SM na população adulta brasileira, e por Mota Júnior et al. (2020), quando analisaram a prevalência desta síndrome em professores da educação básica da rede privada do município de Viçosa-MG. Isso pode ser justificado na literatura, que evidencia que normalmente as mulheres são mais propensas que os homens a buscar por serviços de saúde (Pinheiro \& Couto, 2013).

No estudo de Portugal et al. (2019), a faixa etária mais frequente foi de 31 a 40 anos, em que os autores avaliaram o estado nutricional e a SM em adultos que frequentavam uma UBS em Anajás - PA. Souza et al. (2015), ao analisarem a prevalência da obesidade e da SM em frequentadores de um parque ao ar livre em Recife - PE, verificaram que nesse grupo a faixa de idade predominante foi de 50 a 59 anos, assemelhando-se a do presente estudo. Portugal et al. (2019), encontraram em sua amostra predominância de ensino fundamental completo e renda de 1 a 2 Sm. Enquanto, no estudo de Souza et al. (2015), a maioria foi entre aqueles que concluíram o ensino superior, e a renda mensal era entre 4 a $10 \mathrm{Sm}$. As características da amostra estudada por Souza et al. (2015), tem maior semelhança com a deste estudo, do que aquelas encontradas por Portugal et al. (2019). Quanto à etnia autorrelatada pelos servidores deste trabalho, o predomínio foi entre os pardos, assim como no estudo feito por Leão (2013). Já no estudo de Vieira et al. (2014), realizado em Goiânia-GO a maior frequência foi de brancos, divergindo de nossos achados.

Dentre os componentes da SM preconizados pela IDBSM e pelo NCEP-ATP III, o HDL-c baixo, seguido pela OC e PA elevada, foram os fatores de risco para SM mais frequentes na amostra deste estudo. Entre estes componentes, o HDL-c baixo e a OC, tiveram uma prevalência estatisticamente significante, sendo similares aos encontrados por Mota Júnior et al. (2020), e Ito et al. (2014). Nesses estudos, os componentes da SM de maior prevalência foram, respectivamente, OC, HDL-c baixo e PA elevada; e OC, HDL-c baixo e TG elevados. A maior prevalência destes dois componentes da SM na amostra estudada lhe confere maior risco de desenvolvimento de um evento cardiovascular (Andrade et al., 2013; Cesarino et al., 2013; Martini et al., 2014; Azambuja et al., 2015).

Quando a avaliação dos constituintes da SM foi de acordo com o sexo, o HDL-c baixo foi o mais prevalente em ambos, seguido da OC entre as mulheres, e da PA elevada entre os homens. Estes dados são semelhantes aos de Oliveira et al. (2020). Em 2016, Bortoletto et al. investigou na população de Cambé - PR, entre as pessoas com 40 anos ou mais, a prevalência de SM, de seus componentes e fatores relacionados. Entre os homens, os componentes mais frequentes foram PA, TG e glicose elevados. Nas mulheres foram mais proeminentes OC, PA elevada e HDL-c baixo. A PA elevada com maior frequência nos homens é relacionada a elementos biológicos e comportamentais (Silva et al., 2016; Mota Júnior et al., 2020). Nas mulheres, a OC e HDLc baixo, sendo os componentes da SM com maior percentual chamam atenção, principalmente, naquelas que se encontram no 
período de transição, na menopausa, ou pós-menopausa, caracterizado por alterações hormonais e pela androgenia, que provoca a perda do efeito protetor do estrogênio, e influenciam no ganho de peso e alterações no perfil lipídico aumentando o risco de desenvolvimento da SM (Veloso et al., 2014).

$\mathrm{Na}$ análise do número de componentes simultâneos da SM, verificou-se que o percentual de participantes com a agregação de todos os componentes foi menor que daqueles que não continham nenhum componente. Entretanto, a grande maioria desta amostra apresentava componentes da SM, perfil similar ao encontrado na população brasileira adulta (Oliveira et al., 2020). No presente estudo a frequência de homens com a agregação de 5 elementos, foi o triplo da verificada nas mulheres. $\mathrm{O}$ agrupamento de 3 componentes foi mais prevalente entre eles, e de 2 componentes entre elas. Neste sentido, vale salientar que Klein et al. (2002), ao avaliarem o risco de desenvolvimento de DCVs, relacionaram o número de componentes para SM e concluíram que o risco de desenvolver DCVs foi seis vezes maior quando quatro ou mais componentes estavam presentes.

Neste estudo, aproximadamente metade dos servidores se encaixou nos critérios preconizados pela I DBSM e pelo NCEP-ATP III, sendo assim, classificados em "Presença de SM", prevalência maior do que a verificada em brasileiros adultos 38,4\%, (Oliveira et al., 2020); em professores da rede privada 28,7\%, (Mota Júnior et al., 2020); em anajaenses adultos 7,5\%, (Portugal et al., 2019); e em frequentadores de um parque em Recife-PE 4,3\% (Souza et al., 2015). No entanto, foi verificada maior prevalência da SM do que a deste estudo, entre os cambeenses com 40 anos ou mais 53,7\%, (Bortoletto et al., 2016); entre portadores de SM avaliados por diferentes critérios, segundo o NCEP-ATP III, 57,6\% (Azambuja et al., 2015), e em adultos com DCNTs atendidos no Hospital Universitário João de Barros Barreto (HUJBB), Belém-PA 72,86\%, (Pinho et al., 2014). Apesar do importante percentual de servidores classificados com a "Presença de SM" nesta análise não houve significância estatística.

As inconformidades destes resultados, possivelmente podem ter ocorrido devido a pluralidade nacional presente nas diferenças regionais, culturais e costumais, que afetam o perfil populacional, e também devido às diferentes amostras estudadas: servidores públicos, profissionais da educação privada e adultos de modo geral. Outro fator contribuinte para essa discrepância, percebida até nos estudos realizados no mesmo município, foi o uso de diversos métodos, que adotaram critérios distintos, para determinar a presença da SM. Além do que a estimativa dos componentes da SM, pode ocorrer pela utilização de dados aferidos e autorrelatados. Deste modo, as prevalências da SM e de seus componentes como, a dos fatores bioquímicos, quando autorrelatados podem ser subestimadas (Pinho et al., 2014; Bortoletto et al., 2016; Portugal et al., 2019; Mota Júnior et al., 2020; Oliveira et al., 2020).

Entre os critérios estabelecidos pela I DBSM e pelo NCEP-ATP III para a definição da SM, estão os componentes do perfil lipídico, HDL-c e TG, e o índice glicêmico (Ford, 2005; Carvalho, 2005). No presente estudo a avaliação destes componentes foi realizada por meio de coleta sanguínea para a execução dos testes bioquímicos, o que pode ter contribuído para a importante prevalência de SM encontrada entre os servidores técnicos. Pois o uso de dados laboratoriais permite que se obtenha resultados fiéis à condição de saúde do indivíduo, e possibilitam avaliar a prevalência de danos à saúde em estágios iniciais, subclínicos, sem diagnóstico e tratamento. A identificação de distúrbios cardiometabólicos em tempo oportuno permite a prevenção de eventos mais graves como a SM (Lee et al., 2017; Wang et al., 2020).

Segundo Pinho et al. (2014), o ERF apresenta custo baixo, boa confiabilidade, é simples e possibilita a execução de rastreamento rigoroso, além de indicações terapêuticas mais assertivas para futuros casos de eventos cardiovasculares. Por isso, é um dos métodos mais utilizados para identificação de pacientes ambulatoriais sob maior risco de desenvolver DCVs. Estudos epidemiológicos confirmam que na ausência dos fatores de risco, as DCVs seriam uma causa relativamente rara de morte. Por isso, a prevenção primária com rastreamento, detecção e manuseio desses fatores é a grande aposta para evitar ou reduzir a progressão das DCVs e de suas complicações de forma menos nociva e eficaz (Gomes et al., 2012). Estudos de monitoramento de fatores de risco para DCNTs tornam-se importantes no desenvolvimento, apoio e avaliação de políticas de promoção à saúde e enfrentamento dessas doenças (Malta \& Silva Junior, 2013). Partindo deste pressuposto, neste estudo a maioria dos 
participantes apresentaram baixo risco para desenvolvimento de eventos cardiovasculares, entretanto o quantitativo classificado com alto risco de desenvolver um evento cardiovascular em 10 anos foi elevado.

$\mathrm{Na}$ estratificação do risco cardiovascular pelo ERF, ao relacioná-lo com a presença ou ausência da SM, verificamos que no estrato de baixo risco, o maior percentual obtido foi entre os classificados com "Ausência de SM". Todavia, com relação ao risco de eventos cardiovasculares e a "Presença de SM", nos estratos de médio e alto risco do ERF, o maior percentual foi de servidores classificados com "Presença de SM", assemelhando-se aos resultados de Pinho et al. (2014). Oliveira et al. (2014) estimaram que no escore de Framingham, a SM prenuncia um risco de 25\% para o desenvolvimento de um evento cardiovascular em 10 anos. Para Carvalho (2005), e Ferreira et al. (2013) a correlação da SM com o estrato de alto risco do ERF, prediz o aumento da taxa de mortalidade cardiovascular. Neste estudo observou-se que a maior prevalência de "Presença de SM" foi diretamente relacionada com maior risco cardiovascular, nesta análise houve diferença significativa. $\mathrm{O}$ risco relativo entre estas variáveis mostrou que naqueles com "Presença de SM" o risco de desenvolver um evento cardiovascular é aproximadamente 3,2 vezes maior do que entre aqueles classificados com "Ausência de SM", maior do que o encontrado por Simão et al. (2013).

O aumento da prevalência dos componentes da SM neste trabalho foi diretamente proporcional à elevação da idade, relação estatisticamente significativa, corroborando com os achados de Bortoletto et al. (2016), Portugal et al. (2019), e Oliveira et al. (2020). Entretanto, aqueles pertencentes ao grupo de menor faixa etária também apresentaram uma importante frequência destes componentes, com destaque para o HDL-c baixo e para a OC. A "Presença de SM" foi percebida em quase 20\% deles. Estes resultados demonstram que a prevalência destes fatores em pessoas mais jovens aumenta o risco de desenvolvimento precoce de SM. Além do que, estes componentes também são constituintes das DCVs e tem importante associação a níveis mais altos de mortalidade, até mesmo em adultos jovens (Pinho et al., 2014; Souza et al., 2015).

Na faixa etária intermediária a maior parte se encaixava nos critérios de definição da SM, em que apenas a glicose e os TG elevados tiveram prevalência menor que 50\%. Entre os servidores com mais de 60 anos, destaca-se a elevada frequência de todos os componentes desta síndrome, especialmente da PA e do HDL-c. Neste recorte a "Presença de SM" foi mostrada na grande maioria deles. Nos estudos de Vieira et al. (2014), e Santos et al. (2017), que buscaram estimar a frequência da SM e dos fatores relacionados em idosos usuários do Sistema Único de Saúde em Goiânia-GO e em Belém-PA, verificaram que a prevalência da SM foi menor do que a encontrada neste estudo no grupo com idade superior a 60 anos.

Entre a população sênior a incidência de HAS e a prevalência de níveis pressóricos aumentados estão propensas a elevação. O HDL-c em níveis adequados tem ação cardioprotetora, mas com índices inadequados apresentam redução da proteção cardiovascular (Vieira et al., 2014). Sendo assim, a alta prevalência destes componentes e da "Presença de SM" nos servidores seniores chamam atenção, sobretudo pela intrínseca relação da SM com as DCNTs, que comprometem o funcionamento do sistema cardiovascular e consequentemente aumentam o risco da morbimortalidade cardiovascular (Santos et al., 2017; Zoraski et al., 2017).

Diversos elementos são considerados determinantes da saúde, como os comportamentais, socioeconômicos, culturais, étnicos e psicológicos e conforme a forma com a qual as pessoas são expostas a eles podem influenciar no surgimento de complicações, provocando desequilíbrios na sua condição de saúde (Carrapato et al., 2017). Neste contexto, a SM é uma situação de saúde multifatorial, condicionada ao perfil social e econômico abrangendo a etnia, renda e escolaridade (Guallar-Castillón et al., 2014; Cho et al., 2016; Pucci et al., 2017).

$\mathrm{Na}$ avaliação da prevalência da "Presença de SM" relacionada aos determinantes de saúde, raça/etnia, escolaridade e renda, houve predominância entre as pessoas de pele preta, seguida pelos pardos e pelas de pele branca. No estudo de Vieira et al. (2014), e de Oliveira et al. (2020) não houve diferença significativa da prevalência da SM entre os grupos étnicos. Para Gronner et al. (2011) houve associação significativa da prevalência da SM e a cor de pele branca. 
Quanto a correlação dos componentes da SM com a raça/etnia, vários autores descrevem que a morbimortalidade consequente da HAS e dos demais componentes da SM é mais prevalente em pessoas de cor de pele preta (Lotufo \& Benseno, 2013; Silveira et al., 2013; Barroso et al., 2020). Segundo Singh et al. (2012) e Menni et al. (2013) o estilo de vida e componentes sociais e econômicos tem maior impacto sobre as diferentes prevalências encontradas entre as pessoas de cor de pele preta, parda e branca. Neste estudo os níveis pressóricos elevados foram mais frequentes entre os servidores de cor de pele preta, entretanto não houve diferença significativa quando comparado aos pardos e brancos, assim como no inquérito Vigitel 2018 (MS, 2019) e no estudo realizado por Andrade et al. (2015). Estes achados podem ser justificados devido a associação entre os componentes da SM e a raça/etnia ser influenciada pela inter-relação das desigualdades socioeconômicas e dos fatores hereditários que refletem diretamente na adoção de hábitos benéficos ou nocivos à saúde. Evidenciando que em grupos populacionais com menor recursos financeiros a frequência de morbidade causadas pelos componentes da SM é maior (Lotufo \& Benseno, 2013; Selem et al., 2013).

Na relação com a escolaridade percebeu-se que a elevação da prevalência da "Presença de SM", foi inversamente proporcional ao nível de escolaridade, perfil semelhante ao encontrado por Souza et al. (2015); Bortoletto et al. (2016); Zoraski et al. (2017); Santos et al. (2017); e Oliveira et al. (2020). Quanto a análise da renda mensal dos servidores foi percebida maior prevalência da "Presença de SM" naqueles com as maiores faixas salariais divergindo do estudo de Sarrafzadegan et al. (2011), onde foi observado maior frequência de SM no grupo com menor recursos financeiros.

Neste estudo a avaliação dos determinantes socioeconômicos relacionados à "Presença de SM" demonstrou que não houve diferença significativa, corroborando com os achados de Gronner et al. (2011). A SM tem forte ligação com estes determinantes, que têm influência sobre hábitos de vida nocivos, hábitos alimentares e a prática de exercícios físicos. Fatores que podem estar associados ao desenvolvimento e agrupamento das DCNTs e de morbimortalidade precoce (Ramires et al., 2018). Assim, estes resultados certificam a opinião de outros pesquisadores, ao inferir a condição socioeconômica como dado de risco modificável através de estratégias e políticas públicas locais e globais de saúde (Stringhini et al., 2017).

Diante desse contexto, é primordial a elaboração de ações de promoção à saúde e prevenção de doenças por meio da identificação precoce dos fatores de risco para o desenvolvimento da SM e das DCVs. Ademais, estes serviços precisam ser acessíveis a toda sociedade, principalmente aqueles mais expostos a esses riscos (Simão et al., 2013; Malachias et al., 2016). Um estudo produzido por Cazarim et al. (2016), mostrou que a prestação de serviços farmacêuticos é um método profissional possível de ser implantado nos serviços de saúde, e que contribuiu para favorecer o controle da PA e do colesterol, proporcionou o alcance da adesão ao medicamento, o aumento dos cuidados preventivos e a redução do risco cardiovascular, além de minimizar a morbimortalidade por HAS e promoveu a melhora na qualidade de vida dos usuários, resultados que permaneceram por pelo menos três anos após a alta dos participantes.

Deste modo o farmacêutico como promotor de saúde na atenção básica, seja na rede pública, inserido na equipe multiprofissional, ou na rede privada tem posição estratégica e pode ser protagonista nas ações de prevenção, educação, promoção e melhora da qualidade de vida da comunidade, devido ao elevado percentual de pessoas com quem tem contato diariamente por conveniência. Sendo assim o farmacêutico torna-se um profissional de saúde essencial no rastreamento dos fatores de risco para prevenção da SM (MS, 2014; CFF, 2016).

A oferta do rastreamento em saúde por farmacêuticos aos servidores técnicos, neste trabalho, revelou um importante índice de participantes propensos ao desenvolvimento de DCNTs, naqueles sem diagnóstico prévio de HAS, DM2 e dislipidemias, e o agravamento destas condições entre os servidores já diagnosticados, mas sem o controle adequado devido a diversos motivos, como hábitos de vida nocivos e uso irracional dos medicamentos, condições que impactam diretamente na vida laboral, e trazem prejuízos a estes servidores através da redução de suas atividades e da sua produtividade, devido a necessidade de afastamento temporário para tratamento. 
O amplo conjunto de atribuições do farmacêutico e sua acessibilidade conferem a este profissional uma colocação estratégica na prestação do serviço de rastreamento em saúde. Este serviço não se trata apenas da aplicação de testes ou questionários, ele também engloba a análise dos resultados em busca da ação mais assertiva conforme a condição de saúde do paciente (CFF, 2016). Durante as ações de rastreamento, o farmacêutico também pôde oferecer aos servidores orientações sobre autonomia de cuidados em saúde, mediante educação em saúde e orientações sobre o uso racional de medicamentos, armazenamento adequado, e ajustes de horários.

Este trabalho foi realizado por meio da parceria do Ambulatório de Cuidados Farmacêuticos com as pró-reitorias e demais órgãos dentro da Universidade que atuam nos serviços de saúde direcionados aos servidores. Realizar ações em saúde para prevenir doenças e agravos e promover saúde e melhora da qualidade de vida destes servidores são essenciais para minimizar o risco de desenvolvimento da SM e de eventos cardiovasculares. A SM tem sido bastante pesquisada em vários grupos populacionais, entretanto foram escassos os estudos que avaliaram esta síndrome entre servidores técnicos de Universidades Públicas, ressaltando a importância deste trabalho.

Os achados deste estudo, revelaram um elevado percentual de servidores classificados em "Presença de SM", com maior prevalência entre as mulheres. Também foi possível detectar nesta amostra, uma alta frequência dos componentes da SM, com maior percentagem do HDL-c baixo, OC e PA elevada. O HDL-c baixo e a OC foram mais prevalentes entre as mulheres e a PA elevada foi mais prevalente entre os homens. A relação da "Presença de SM" com os estratos de risco do ERF e com as faixas etárias foi diretamente proporcional.

\section{Conclusão}

O rastreamento em saúde realizado pelo farmacêutico demonstrou-se eficaz no que se refere à identificação de possíveis casos de SM e de seus componentes. A prestação deste serviço por este profissional permitiu também a oferta da educação em saúde por meio do acesso a informações e orientação acerca de medidas para prevenção e controle das DCNTs que influenciarão positivamente os servidores técnicos na adesão ao tratamento farmacológico e na adoção de medidas não farmacológicas como a mudança de estilo de vida para favorecer a melhora da qualidade de vida e consequentemente reduzir o risco do desenvolvimento da SM e das DCVs. Os resultados deste estudo demonstraram a necessidade no ambiente universitário de realizar ações de promoção em saúde, por meio da implantação de serviços de saúde e de uma equipe multiprofissional.

Vale ressaltar a importância destes resultados e a necessidade de considerá-los nas discussões sobre promoção de saúde do servidor, com o intuito de estabelecer medidas de prevenção, controle e tratamento da SM e das DCVs neste grupo. Além de embasar a elaboração de outros estudos acerca do tema, com maior abrangência e maior número amostral que possam corroborar com estes achados.

\section{Agradecimentos}

Agradecimentos à Universidade Federal do Pará por meio da Pró-Reitoria de Desenvolvimento e Gestão de Pessoal (PROGEP), do Programa de Pós-Graduação em Assistência Farmacêutica (PPGAF) e do Núcleo de Excelência em Cuidados Farmacêuticos (NECFAR), pelo apoio ao desenvolvimento desta pesquisa.

\section{Referências}

Ambiel, I. S. S., \& Mastroianni, P. D. C. (2013). Resultados da atenção farmacêutica no Brasil: uma revisão. Revista de Ciências Farmacêuticas Básica e Aplicada, 34(4), 469-474. https://repositorio.unesp.br/handle/11449/133737

Andrade, J. P. D., Mattos, L. A. P., Carvalho, A. C., Machado, C. A., \& Oliveira, G. M. M. D. (2013). Programa nacional de qualificação de médicos na prevenção e atenção integral às doenças cardiovasculares. Arquivos Brasileiros de Cardiologia, 100(3), 203-211. https://doi.org/10.5935/abc.20130061 
Andrade, S. S. D. A., Stopa, S. R., Brito, A. S., Chueri, P. S., Szwarcwald, C. L., \& Malta, D. C. (2015). Prevalência de hipertensão arterial autorreferida na população brasileira: análise da Pesquisa Nacional de Saúde, 2013. Epidemiologia e Serviços de Saúde, 24(2), 297-304. https://doi.org/10.5123/S167949742015000200012

Associação Brasileira para o Estudo da Obesidade e da Síndrome Metabólica. (2016). VI Diretrizes Brasileiras de Obesidade, (4a ed.). https://abeso.org.br/wpcontent/uploads/2019/12/Diretrizes-Download-Diretrizes-Brasileiras-de-Obesidade-2016.pdf

Azambuja, C. R., Farinha, J. B., Rossi, D. S., Spohr, C. F., \& dos Santos, D. L. (2015). O Diagnóstico da síndrome metabólica analisado sob diferentes critérios de definição. Revista Baiana de Saúde Pública, 39(3), 482-482. https://doi.org/10.22278/2318-2660.2015.v39.n3.a728

Barros, D. S. L., Silva, D. L. M., \& Leite, S. N. (2019). Serviços farmacêuticos clínicos na atenção primária à saúde do Brasil. Trabalho, Educação e Saúde, 18, 1-17. https://doi.org/10.1590/1981-7746-sol00240

Barroso, W. K. S., Rodrigues, C. I. S., Bortolotto, L. A., Mota-Gomes, M. A., Brandão, A. A., Feitosa, A. D. D. M., Machado, C.A., Poli-de-Figueiredo, E.C., Amodeo, C., Mion Júnior, D., Barbosa, E. C. D., Nobre, F., Guimarães, I. C. B., Vilela-Martin, J. F., Yugar-Toledo, J.C., Magalhães, M. E. C., Neves, M. F. T., Jardim, P. C. B. V., Miranda, D. R., \& Nadruz, W. (2021). Diretrizes Brasileiras de Hipertensão Arterial-2020. Arquivos Brasileiros de Cardiologia, 116(3), 516-658. https://doi.org/10.36660/abc.20201238

Bortoletto, M. S. S., Souza, R. K. T. D., Cabrera, M. A. S., \& González, A. D. (2016). Síndrome metabólica, componentes e fatores associados em adultos de 40 anos ou mais de um município da Região Sul do Brasil. Cadernos Saúde Coletiva, 24, 32-40. https://doi.org/10.1590/1414-462X201600010123

Carrapato, P., Correia, P., \& Garcia, B. (2017). Determinante da saúde no Brasil: a procura da equidade na saúde. Saúde e Sociedade, 26(3), 676-689. https://doi.org/10.1590/S0104-12902017170304

Carvalho, M. H. C. (2005). I Diretriz brasileira de diagnóstico e tratamento da síndrome metabólica. Arquivos Brasileiros de Cardiologia, 84, 1-28. http://publicacoes.cardiol.br/consenso/2005/sindromemetabolica.asp

Cazarim, M. D. S., Freitas, O., Penaforte, T. R., Achcar, A., \& Pereira, L. R. L. (2016). Impact assessment of pharmaceutical care in the management of hypertension and coronary risk factors after discharge. PloS one, 11(6),2-14. https://doi.org/10.1371/journal.pone.0155204

Cesarino, C. B., Borges, P. P., Ribeiro, R. D. C. H. M., Ribeiro, D. F., \& Kusumota, L. (2013). Avaliação do risco cardiovascular de pacientes renais crônicos segundo critérios de Framingham. Acta Paulista de Enfermagem, 26, 101-107. https://doi.org/10.1590/S0103-21002013000100016

Cho, K. I., Kim, B. H., Je, H. G., Jang, J. S., \& Park, Y. H. (2016). Gender-Specific associations between socioeconomic status and psychological factors and metabolic syndrome in the Korean population: Findings from the 2013 Korean National Health and Nutrition Examination Survey. BioMed research international. 2016, 1-8. https://doi.org/10.1155/2016/3973197

Cordero, A., Bertomeu-Martínez, V., Mazón, P., Fácila, L., Cosín, J., Bertomeu-González, V., Rodriguez, M., Andrés, E., Galve, E., Lekuona, I., \& GonzálezJuanatey, J. R. (2013). Patients with cardiac disease: Changes observed through last decade in out-patient clinics. World Journal of Cardiology, 5(8), 288-294. https://doi:10.4330/wjc.v5.i8.288

Conselho Federal de Farmácia (2016). Serviços farmacêuticos diretamente destinados ao paciente, à família e à comunidade: contextualização e arcabouço conceitual,1-200.https://www.cff.org.br/userfiles/Profar_Arcabouco_TELA_FINAL.pdf

Faludi, A. A., Izar, M. C. D. O., Saraiva, J. F. K., Chacra, A. P. M., Bianco, H. T., Afiune Neto, A., Bertolami, A., Pereira, A.C., Lottenberg, A. M., Sposito, A. C., Chagas, A. C. P., Casella Filho, A., Simão, A.F., Alencar Filho, A.C., Caramelli, B., Magalhães, C. C., Negrão, C. E., Ferreira, C. E. S., Scher, C... \& Salgado Filho, W. (2017). Atualização da diretriz brasileira de dislipidemias e prevenção da aterosclerose. Arquivos brasileiros de cardiologia, 109(2), 1-76. https://doi.org/10.5935/abc.20170121

Ferreira, K. C. A., Machado, L. M. M., Pires Filho, J. A., \& de Souza Araújo, M. (2013). Associação entre a presença de síndrome metabólica e variáveis antropométricas, clinicobioquímicas e escore de risco cardiovascular em enfermeiras de um hospital universitário. Brasília Med,50(4), 302-311. http://rbm.org.br/export-pdf/110/v50n4a05.pdf

Ford, E. S. (2005). Prevalence of the metabolic syndrome defined by the International Diabetes Federation among adults in the US. Diabetes care, 28(11), 27452749. https://doi.org/10.2337/diacare.28.11.2745

Golbert, A., Rocha, A. M., Vasques, A. C. J., Ribeiro, A. L. C. P., Vianna, A. G. D., Bauer, A. C., Lerário, A. C., Pires, A. C., Bordon, A. F., Tschiddel, B., Wajchenberg, B. L., Geloneze, B., Negrato, C. A., Couri, C. E. B., Ponte, C. M. M., Pieper, C. M., Leitão, C. B., Valerio, C., Gadelha, D. D., \& Fernandes, V. O. (2017). Diretrizes da Sociedade Brasileira de Diabetes 2017-2018. São Paulo: Editora Clannad, 91. https://diabetes.org.br/e-book/diretrizes-da-sociedadebrasileira-de-diabetes-2017-2018/

Gomes, E. B., Moreira, T. M. M., Pereira, H. C. V., Sales, I. B., Lima, F. E. T., Freitas, C. H. A. D., \& Rodrigues, D. P. (2012). Fatores de risco cardiovascular em adultos jovens de um município do Nordeste brasileiro. Revista Brasileira de Enfermagem, 65(4), 594-600. https://doi.org/10.1590/S003471672012000400007

Gronner, M. F., Bosi, P. L., Carvalho, A. M., Casale, G., Contrera, D., Pereira, M. A., Diogo, T. M., Torquato, M. T. C. G., Souza, G. M. D., Oishi, J., \& Leal, A. M. O. (2011). Prevalence of metabolic syndrome and its association with educational inequalities among Brazilian adults: a population-based study. Brazilian Journal of Medical and Biological Research, 44(7), 713-719. https://doi.org/10.1590/S0100-879X2011007500087

Guallar-Castillón, P., Pérez, R. F., García, E. L., León-Muñoz, L. M., Aguilera, M. T., Graciani, A., Gutiérrez-Fisac, J.L., Banegas, J.R., \& Rodríguez-Artalejo, F. (2014). Magnitude and management of metabolic syndrome in Spain in 2008-2010: the ENRICA study. Revista Española de Cardiología (English Edition), 67(5), 367-373. https://doi.org/10.1016/j.rec.2013.08.014

Ito, C. A. S., Fagundes, C., Plocharski, M., Maciel, M. A. S., Bail, L., \& Vellosa, J. C. R. (2014). Risco presumido para doenças cardiovasculares em servidores de uma universidade estadual do Paraná, Brasil. Revista de Ciências Farmacêuticas Básica e Aplicada,35(4), 623-631. http://rcfba.fcfar.unesp.br/index.php/ojs/article/view/97 
Junqueira, C. D. L. C., Costa, G. M., \& Magalhães, M. E. C. (2011). O risco cardiovascular é maior que o risco dos seus componentes isoladamente? Revista Brasileira de Cardiologia, 24(5), 308-315. http://sociedades.cardiol.br/socerj/revista/2011_05/2a_2011_v24_n05_07sindrome.pdf

Kaur, J. (2014). A comprehensive review on metabolic syndrome. Cardiology Research and Practice, 2014, 943162-943162. http://dx.doi.org/10.1155/2014/943162

Klein, B. E., Klein, R., \& Lee, K. E. (2002). Components of the metabolic syndrome and risk of cardiovascular disease and diabetes in Beaver Dam. Diabetes care, 25(10), 1790-1794. https://doi.org/10.2337/diacare.25.10.1790

Leão, A. L. M. (2013). Síndrome Metabólica em funcionários de uma universidade pública: fatores associados e concordância entre critérios de diagnóstico. https://pesquisa.bvsalud.org/portal/resource/pt/bde-24991

Lee, J. S., Chang, P. Y., Zhang, Y., Kizer, J. R., Best, L. G., \& Howard, B. V. (2017). Triglyceride and HDL-C dyslipidemia and risks of coronary heart disease and ischemic stroke by glycemic dysregulation status: the strong heart study. Diabetes care, 40(4), 529-537. https://doi.org/10.2337/dc16-1958

Leitão, M. P. C., \& Martins, I. S. (2012). Prevalência e fatores associados à síndrome metabólica em usuários de Unidades Básicas de Saúde em São PauloSP. Revista da Associação Médica Brasileira, 58, 60-69. https://doi.org/10.1590/S0104-42302012000100016

Lotufo, P. A., \& Bensenor, I. J. M. (2013). Raça e mortalidade cerebrovascular no Brasil. Revista de Saúde Pública, 47(6), 1201-1204. https://doi.org/10.1590/S0034-8910.2013047004890

Malachias, M. V. B., Souza, W. K. S. B., Plavnik, F. L., Rodrigues, C. I. S., Brandão, A. A., Neves, M. F. T., Bortolotto, L. A., Franco, R. J. S., Poli-deFigueiredo, C. E., Jardim, P. C. B. V., Amodeo, C., Barbosa, E. C. D., Koch, V., Gomes, M. A. M., Paula, R. B., Póvoa, R. M. S., Colombo, F. C., Ferreira Filho, S., \& Miranda, R. D. (2016). 7ª Diretriz Brasileira de Hipertensão Arterial. Arquivos Brasileiros de Cardiologia, 107(3), 1-83.

Malta, D. C., \& Silva Junior, J. B. D. (2013). O Plano de Ações Estratégicas para o Enfrentamento das Doenças Crônicas Não Transmissíveis no Brasil e a definição das metas globais para o enfrentamento dessas doenças até 2025: uma revisão. Epidemiologia e Serviços de Saúde, 22(1), 151-164. http://dx.doi.org/10.5123/S1679-49742013000100016

Martini, F. A. N., Borges, M. B., \& Guedes, D. P. (2014). Hábito alimentar e síndrome metabólica em uma amostra de adultos brasileiros. Archivos Latinoamericanos de Nutrición, 64(3), 161-173. http://ve.scielo.org/pdf/alan/v64n3/art03.pdf

Menni, C., Mangino, M., Zhang, F., Clement, G., Snieder, H., Padmanabhan, S., \& Spector, T. D. (2013). Heritability analyses show visit-to-visit blood pressure variability reflects different pathological phenotypes in younger and older adults: evidence from UK twins. Journal of hypertension, 31(12), 2356-2361. http://dx.doi.org/10.1097/HJH.0b013e32836523c1

Ministério da Saúde. (2010). Rastreamento: normas e manuais técnicos, http://189.28.128.100/dab/docs/publicacoes/cadernos_ab/abcad29.pdf

Ministério da Saúde. (2011). Orientações para coleta e análise de dados antropométricos em serviços de saúde: norma técnica do sistema de Vigilância Alimentar e Nutricional-SISVAN. https://bvsms.saude.gov.br/bvs/publicacoes/orientacoes_coleta_analise_dados_antropometricos.pdf

Ministério da Saúde. (2014). Capacitação para implantação dos serviços de clínica farmacêutica. https://bvsms.saude.gov.br/bvs/publicacoes/cuidado_farmaceutico_atencao_basica_saude_2.pdf

Ministério da Saúde. (2019). Vigitel Brasil 2018: vigilância de fatores de risco e proteção para doenças crônicas por inquérito telefônico: estimativas sobre frequência e distribuição sociodemográfica de fatores de risco e proteção para doenças crônicas nas capitais dos 26 estados brasileiros e no Distrito Federal em 2018. https://portalarquivos2.saude.gov.br/images/pdf/2019/julho/25/vigitel-brasil-2018.pdf

Mota Júnior, R. J., Oliveira, R. A. R., Lima, L. M., Franceschini, S. D. C. C., \& Marins, J. C. B. (2020). Síndrome Metabólica e sua associação com fatores de risco cardiovascular em professores. RBONE-Revista Brasileira de Obesidade, Nutrição e Emagrecimento, 14(86), 467-476. http://www.rbone.com.br/index.php/rbone/article/view/1296

Oliveira, R. P. M., Sérvio, M. T. C., \& Barbieri, M. L. G. (2014). Análise da prevalência de associação da síndrome metabólica com o aparecimento de doenças cardiovasculares. https://www.efdeportes.com/efd195/sindrome-metabolica-com-doencas-cardiovasculares.htm

Oliveira, L. V. A., Santos, B. N. S. D., Machado, Í. E., Malta, D. C., Velasquez-Melendez, G., \& Felisbino-Mendes, M. S. (2020). Prevalência da Síndrome Metabólica e seus componentes na população adulta brasileira. Ciência \& Saúde Coletiva, 25, 4269-4280. https://doi.org/10.1590/1413812320202511.31202020

Peeling, R.W., \& Ye, H. (2004). Diagnostic tools for preventing and managing maternal and congenital syphilis: an overview. Bulletin of the World Health Organization, 82(6), 439-446. https://www.scielosp.org/article/ssm/content/raw/?resource_ssm_path=/media/assets/bwho/v82n6/v82n6a10.pdf

Pinheiro, T. F., \& Couto, M. T. (2013). Sexualidade e reprodução: discutindo gênero e integralidade na Atenção Primária à Saúde. Physis: Revista de Saúde Coletiva, 23, 73-92. http://observatorio.fm.usp.br/handle/OPI/5836

Pinho, C. P. S., Diniz, A. D. S., Arruda, I. K. G. D., Batista Filho, M., Coelho, P. C., Sequeira, L. A. D. S., \& Lira, P. I. C. D. (2013). Prevalência e fatores associados à obesidade abdominal em indivíduos na faixa etária de 25 a 59 anos do Estado de Pernambuco, Brasil. Cadernos de Saúde Pública, 29(2), 313-324. https://doi.org/10.1590/S0102-311X2013000200018

Portugal, P. R., Miranda, R. D. N. A., Ataíde, B. R. B., \& Bandeira, C. C. (2019). Estado nutricional e síndrome metabólica em adultos de uma unidade de saúde de Anajás/PA. Nutrição Brasil, 18(2), 80-87. https://doi.org/10.33233/nb.v18i2.3641

Précoma, D. B., Oliveira, G. M. M. D., Simão, A. F., Dutra, O. P., Coelho, O. R., Izar, M. C. D. O., Póvoa, R. M. S., Giuliano, I. C. B., Alencar Filho, A. C., Machado, C. A., Scherr, C., Fonseca, F. A. H., Santos Filho, R. D., Carvalho, T., Avezum Júnior, A., Esporcatte, R., Nascimento, B. R., Brasil, D. P., Porto, G., . . . \& Mourilhe-Rocha, R. (2019). Atualização da diretriz de prevenção cardiovascular da Sociedade Brasileira de Cardiologia-2019. Arquivos Brasileiros de Cardiologia, 113(4), 787-891. https://doi.org/10.5935/abc.20190204 
Pucci, G., Alcidi, R., Tap, L., Battista, F., Mattace-Raso, F., \& Schillaci, G. (2017). Sex-and gender-related prevalence, cardiovascular risk and therapeutic approach in metabolic syndrome: A review of the literature. Pharmacological research, 120, 34-42. https://doi.org/10.1016/j.phrs.2017.03.008

Ramires, E. K. N. M., Menezes, R. C. E., Longo-Silva G., Santos, T. G., Marinho, P. M., Silveira, J. A. C. (2018). Prevalence and Factors Associated with Metabolic Syndrome among Brazilian Adult Population: National Health Survey -2013. Arquivos Brasileiros de Cardiologia, 110(5), 455-66. https://doi.org/10.5935/abc.20180072

Rubbo-Blanco, M. L., Oliveira, B. N. D., Siqueira Filho, A. G. D., Luiz, R. R., \& Lima, R. D. S. L. (2015). Síndrome metabólica é o principal preditor de isquemia miocárdica na SPECT. Internacional Journal of Cardiovascular Sciences, 28(3), 189-199. https://doi.org/10.5935/2359-4802.20150028

Santos, P. C. M., Ferreira, A. L. L., \& Mori, R. M. S. C. (2017). Frequência da Síndrome Metabólica em idosos cadastrados no Programa Saúde do Idoso de uma Unidade Municipal de Saúde de Belém-PA. Revista da Associação Brasileira de Nutrição, 8, 75-81. https://www.rasbran.com.br/rasbran/article/view/338

Sarrafzadegan, N., Gharipour, M., Ramezani, M.A., Rabiei, K., \& Zolfaghar, B., Tavassoli, A.A., Boshtam, M., Zarfeshani, S., Khosravi, A., \& Yousefi, A. (2011). Metabolic syndrome and health-related quality of life in Iranian population. Journal of Research in Medical Sciences, $16(3), 254-261$. https://www.ncbi.nlm.nih.gov/pmc/articles/PMC3214330/

Selem, S. S. A. D. C., Castro, M. A., César, C. L. G., Marchioni, D. M. L., \& Fisberg, R. M. (2013). Validade da hipertensão autorreferida associa-se inversamente com escolaridade em brasileiros. Arquivos Brasileiros de Cardiologia, 100, 52-59. https://doi.org/10.1590/S0066-782X2012005000119

Silva, E. C., Martins, M. S. A. S., Guimarães, L.V., Segri, N.J., Lopes, M. A. L., \& Espinosa, M. M. (2016). Prevalência de hipertensão arterial sistêmica e fatores associados em homens e mulheres residentes em municípios da Amazônia Legal. Revista Brasileira de Epidemiologia,19, 38-51. https://doi.org/10.1590/1980-5497201600010004

Silveira, J. D., Scherer, F., Deitos, A., \& Bosco, S. M.D. (2013). Fatores associados à hipertensão arterial sistêmica e ao estado nutricional de hipertensos inscritos no programa Hiperdia. Cadernos Saúde Coletiva, 21(2), 129-134. https://pesquisa.bvsalud.org/portal/resource/pt/lil-682410

Simão, A. F., Precoma, D. B., Andrade, J. P., Correa Filho, H., Saraiva, J. F. K., Oliveira, G. M. M., Murro, A. L. B., Campos, A., Alessi, A., Avezum Junior, A., Achutti, A. C., Miguel, A. C. M. G., Sousa, A. C. S., Lotemberg, A. M. P., Lins, A. P., Falud, A. A., Brandão, A. A., Sanjuliani, A. F., Sbissa, A. S., . . \& Souza, W. K. S. B. (2013). I Diretriz brasileira de prevenção cardiovascular. Arquivos brasileiros de cardiologia, 101(6), 163. https://doi.org/10.5935/abc.2013S012

Singh, G. M., Danaei, G., Pelizzari, P. M., Lin, J. K., Cowan, M. J., Stevens, G. A., Farzadfar, F., Khang, Y. -H., Lu, Y., Riley, L. M., Lim, S. S., \& Ezzati, M. (2012). The age associations of blood pressure, cholesterol, and glucose: analysis of health examination surveys from international populations. Circulation, 125(18), 2204-2211. https://doi.org/10.1161/CIRCULATIONAHA.111.058834

Shen, C., Zhou, Z., Lai, S., Tao, X., Zhao, D., Dong, W., Li, D., Lan, X., \& Gao, J. (2019). Urban-rural-specific trend in prevalence of general and central obesity, and association with hypertension in Chinese adults, aged 18-65 years. BMC Public Health, 19 (661), 1-8. https://doi.org/10.1186/s12889-019-7018-4

Soares, T. S., Piovesan, C. H., Gustavo, A. D. S., Macagnan, F. E., Bodanese, L. C., \& Feoli, A. M. P. (2014). Hábitos alimentares, atividade física e escore de risco global de Framingham na síndrome metabólica. Arquivos Brasileiros de Cardiologia, 102(4), 374-382. https://doi.org/10.5935/abc.20140029

Souza, M. D. G. D., Vilar, L., Andrade, C. B. D., Albuquerque, R. D. O., Cordeiro, L. H. D. O., Campos, J. M., \& Ferraz, Á. A. B. (2015). Prevalência de obesidade e síndrome metabólica em frequentadores de um parque. Arquivos Brasileiros de Cirurgia Digestiva, 28, 31-35. https://doi.org/10.1590/S0102$6720201500 \$ 100010$

Stringhini, S., Carmeli, C., Jokela, M., Avendaño, M., Muennig, P., Guida, F., Ricceri, F., d'Errico, A., Barros, H., Bochud, M., Chadeau-Hyam, M., ClavelChapelon, F., Costa, G., Delpierre, C., Fraga, S., Goldberg, M., Giles, G. G., Krogh, V., Kelly-Irving, M., . . \& \& Kivimäki, M. (2017). Socioeconomic status and the $25 \times 25$ risk factors as determinants of premature mortality: a multicohort study and meta-analysis of 1. 7 million men and women. The Lancet, 389(10075), 1229-1237. https://doi.org/10.1016/S0140-6736(16)32380-7

Pinho, P. M., Machado, L. M. M., Torres, R. S., Carmin, S. E. M., Mendes, W. A. A., Silva, A. C. M., Araújo, M. S., \& Ramos, E. M. L. S. (2014). Síndrome metabólica e sua relação com escores de risco cardiovascular em adultos com doenças crônicas não transmissíveis. Revista da Sociedade Brasileira de Clínica Médica, 12, 22-30. https://pesquisa.bvsalud.org/portal/resource/pt/lil-707347

Tsuyuki, R. T., Al Hamarneh, Y. N., Jones, C. A., \& Hemmelgarn, B. R. (2016). The effectiveness of pharmacist interventions on cardiovascular risk: the multicenter randomized controlled RxEACH trial. Journal of the American College of Cardiology, 67(24), 2846-2854. http://dx.doi.org/10.1016/j.jacc.2016.03.528

Veloso, G. G. V., David, A. L. S., Pereira, A. C., Almeida, J. S., Carmo, R. B., Freitas, R. F., Reis, V. M. C. P., Passos, B. M. A., Borges, M. A. R., \& Rocha, J. S. B. (2014). Prevalência de síndrome metabólica em mulheres climatéricas. Revista Brasileira de Cardiologia,27, $20-27$. http://www.onlineijcs.org/english/sumario/27/27-1/artigo2.asp

Vieira, E. C., Peixoto, M. D. R. G., \& Silveira, E. A. D. (2014). Prevalência e fatores associados à Síndrome Metabólica em idosos usuários do Sistema Único de Saúde. Revista Brasileira de Epidemiologia, 17(4), 805-817. https://doi.org/10.1590/1809-4503201400040001

Wang, A., Li, H., Yuan, J., Zuo, Y., Zhang, Y., Chen, S., Wu, S., \& Wanga, Y. (2020). Visit-to-visit variability of lipids measurements and the risk of stroke and stroke types: a prospective cohort study. Journal of stroke, 22, 119-129. https://doi.org/10.5853/jos.2019.02075

Wu, H. F., Tam, T., Jin, L., Lao, X. Q., Chung, R. Y. N., Su, X. F., \& Zee, B. (2017). Age, gender, and socioeconomic gradients in metabolic syndrome: biomarker evidence from a large sample in Taiwan, 2005-2013. Annals of epidemiology, 27(5), 315-322. https://doi.org/10.1016/j.annepidem.2017.04.003

Zoraski, H., Fiametti, M., Santos, R., Gregoletto, M. L. O., \& Cremonese, C. (2017). Síndrome metabólica em idosos de Nova Roma do Sul, RS: prevalência e fatores associados. Arquivos Brasileiros de Ciências da Saúde, 42(3), 147-155. http://dx.doi.org/10.7322/abcshs.v42i3.955 